\title{
EL MERCADO GLOBAL DE APUESTAS DEPORTIVAS ONLINE Terreno fértil para fraudes y crímenes
}

\author{
Marcelo Moriconi \\ Iscte - Instituto Universitário de Lisboa, Centro de Estudos Internacionais (CEI-Iscte), Lisboa, \\ Portugal \\ João Paulo Almeida \\ Comité Olímpico de Portugal, Lisboa, Portugal
}

Resumen Las nuevas tecnologías han modificado radicalmente la industria de las apuestas deportivas. En la actualidad, millones de personas apuestan en línea y en tiempo real, en una amplia gama de eventos que se desarrollan por todo el mundo. Este mercado global ha generado diversas oportunidades para el crimen y el fraude. En el panorama actual, el amaño de partidos ha evolucionado de ser un fenómeno histórico dentro del mundo de los deportes a un problema criminal global que involucra a organizaciones criminales transnacionales, a jugadores profesionales, a empresarios, árbitros, deportistas e intermediarios. A pesar de que la manipulación de competiciones se ha posicionado en la agenda pública como la mayor amenaza contra la sustentabilidad del deporte, la complejidad y extensión del nuevo mercado de apuestas generan áreas de riesgo para el delito que van más allá del simple amaño de partidos. El artículo tiene el objetivo pedagógico y práctico de explicar los cambios en el mercado y tipos de apuestas deportivas y describir las nuevas estructuras de oportunidad para el delito y el fraude que surgen del nuevo marco comercial. Todas estas cuestiones, poco estudiadas, abren una ventana de oportunidad para nuevas líneas de investigación multidisciplinar sobre deporte, criminalidad, economía y políticas públicas.

Palabras-clave: apuestas deportivas, manipulación de partidos, crimen organizado, corrupción, fraude, lavado de dinero.

O mercado global de apostas desportivas online: terreno fértil para a fraude e o crime

Resumo As novas tecnologias mudaram radicalmente a indústria de apostas desportivas. Milhões de pessoas apostam agora online e em tempo real num vasto número de eventos que têm lugar em todo o mundo. Este mercado global criou oportunidades para o crime e a fraude. No contexto atual, a manipulação de resultados evoluiu de um fenómeno histórico do mundo do desporto para um problema criminal global envolvendo organizações criminosas transnacionais, apostadores profissionais, homens de negócios, árbitros, atletas e intermediários. Embora a manipulação de competições se tenha posicionado na agenda pública como a maior ameaça à sustentabilidade do desporto, a complexidade e extensão do novo mercado de apostas gera áreas de risco de criminalidade que vão além da simples manipulação de resultados. O artigo tem por objetivo pedagógico e prático explicar as mudanças no mercado e nos tipos de apostas desportivas, e descrever as novas estruturas de oportunidade para o crime e a fraude que surgem num novo contexto de mercado. Todas estas questões, pouco estudadas, abrem uma janela de oportunidade para novas linhas de investigação multidisciplinar sobre o desporto, a criminalidade, a economia e as políticas públicas.

Palavras-chave: apostas desportivas, manipulação de resultados, crime organizado, corrupção, fraude, lavagem de dinheiro.

Online sports betting market and new technologies: fertile ground for fraud and crime

Abstract The massification of new technologies have radically changed the sports betting industry. Nowadays, millions of people bet online, and in real time, in a wide range of sport events worldwide. This global market has generated several opportunity structures for crime and fraud. In this current landscape, match-fixing has evolved from being a historical phenomenon in the world of sports to a global criminal problem involving transnational criminal syndicates, professional gamblers, businessmen, umpires, athletes, and intermediaries. Despite the fact that match-fixing has been placed on the public agenda as the greatest threat against the 
sustainability of the sport, the complexity and extension of the new betting market generates possibilities for fraud, cheating and crime that go beyond fixed matches or competitions. The article has the pedagogical and practical objective of explaining and systematizing the changes in the betting market and types of bets and describing the new opportunity structures for crime and fraud that arise from the new commercial framework. Considering the lack of studies about these issues, the article opens a window of opportunity for new lines of multidisciplinary research on sport, crime, economics and public policy.

Keywords: sport betting market, match-fixing, organized crime, corruption, fraud, money laundering.

Le marché mondial des paris sportifs en ligne: un terrain fertile pour la fraude et le crime

Résumé Les nouvelles technologies ont radicalement changé l'industrie des paris sportifs. Aujourd'hui, des millions de personnes jouent en ligne et en temps réel sur un large éventail d'événements se déroulant à travers le monde. Ce marché mondial a créé un certain nombre d'opportunités de criminalité et de fraude. Dans le scénario actuel, le trucage de matchs est passé d'un phénomène historique dans le monde du sport à un problème criminel mondial impliquant des organisations criminelles transnationales, des joueurs professionnels, des hommes d'affaires, des arbitres, des athlètes et des intermédiaires. Bien que la manipulation des compétitions se soit positionnée dans l'agenda public comme étant la plus grande menace pour la durabilité du sport, la complexité et l'extension du nouveau marché des paris génère des zones de risque de criminalité au-delà du simple trucage de matchs. Cet article a pour objectif pédagogique et pratique d'expliquer et de systématiser les changements dans le marché et les types de paris sportifs et de décrire les structures d'opportunités pour la criminalité et la fraude qui découlent du nouveau cadre commercial. Toutes ces questions, peu étudiées, peuvent engendrer des opportunités uniques qui pourraient mener à de nouvelles pistes de recherche multidisciplinaire sur le sport, la criminalité, l'économie et les politiques publiques.

Mots-clés: paris sportifs, manipulation de matches, crime organisé, corruption, fraude, blanchiment d'argent.

\section{Introducción}

Gracias a las nuevas tecnologías, el mercado de apuestas deportivas se ha modificado radicalmente y ha conseguido expandirse a una escala global. Actualmente, una persona puede apostar desde Asia por el triunfo de un equipo de fútbol africano que disputa un partido en América Latina a través de un operador de apuestas en línea con sede en Malta (Moriconi y Almeida, 2019b). Además, la transmisión en vivo de eventos deportivos a nivel mundial, junto con la velocidad con la que la información se mueve alrededor del mundo y las infinitas oportunidades de llevar a cabo las apuestas en directo e instantáneamente, han determinado la aparición de nuevos tipos de apuestas, como aquellas relacionadas con la probabilidad de que un evento específico ocurrirá en el transcurso de una competición deportiva. En consecuencia, en tiempo real durante el transcurso de un partido, una persona puede apostar en quién tendrá el próximo saque de esquina en el fútbol, quien ganará el siguiente punto, juego o set en un partido de tenis, quien se llevará la siguiente tarjeta amarilla, o quien marcará el próximo gol. 
Teniendo en cuenta la histórica relación entre el crimen organizado ${ }^{1}$ y el mundo del juego (King, 1969; Huggins, 2018), esta coyuntura ha generado nuevas estructuras de oportunidad para el delito. En los últimos tiempos, y tras diversos escándalos a nivel mundial (Interpol y IOC, 2015), la manipulación de resultados vinculadas a las apuestas ha sido considerada como una de las mayores amenazas para la integridad y sustentabilidad del deporte profesional (Tak, Sam y Jackson, 2018a; Spapens, 2017; Carpenter, 2012; Hill, 2008; Moriconi, 2018a). El problema se convirtió en uno de los puntos clave de las agendas políticas y deportivas. El 1 de septiembre de 2019, por ejemplo, entró en vigor la Convención del Concejo de Europa contra la Manipulación de Competiciones Deportivas (Council of Europe, 2014). La convención, conocida popularmente con el nombre de Macolin, ha sido considerada como la iniciativa política más eficaz para combatir el problema, ya que exige la cooperación entre actores de todos los sectores interesados (organizaciones deportivas y políticas, fuerzas de seguridad, operadores y reguladores de apuestas, etc.) y el establecimiento de un conjunto de recomendaciones prácticas y políticas para la creación y promoción de estructuras institucionales, legales y penales para combatir el flagelo (Serby, 2015; Moriconi, 2020).

A pesar del énfasis de las instituciones políticas y deportivas en la manipulación, el nuevo mercado global de apuestas online genera áreas de riesgo y oportunidades para el delito que van más allá del amaño de partidos y competiciones. En algunos casos, la trampa o el fraude pueden practicarse sin necesidad de manipulaciones. Incluso en algunos momentos, debido a un mercado mundial tan masivo, los apostadores profesionales pueden encontrar errores en el cálculo de las ofertas que permiten colocar apuestas cien por ciento seguras sin necesidad de perpetrar engaño ni cometer ilegalidades.

Si bien el deporte tiene, de acuerdo con la Comisión Europea, una importancia estratégica como factor social, económico y de cooperación (European Commission, 2007; 2011), la relación entre esta actividad, las apuestas y el crimen continúa a ser un tema poco estudiado por las ciencias sociales. En este sentido, este artículo tiene el objetivo pedagógico y práctico de explicar y sistematizar los cambios en el mercado y tipos de apuestas deportivas y enumerar y describir las nuevas estructuras de oportunidad para el delito y el fraude que surgen del nuevo marco comercial. Todas estas cuestiones, poco estudiadas, abren una ventana de oportunidad para nuevas líneas de investigación multidisciplinar sobre deporte, criminalidad, economía y políticas públicas.

1 No es propósito de este trabajo ingresar en el debate académico sobre qué es y qué no es un grupo criminal organizado. Por ello, los autores aclaran que, por crimen organizado, a lo largo de este texto, se entiende lo que define la Convención de las Naciones Unidas contra la Delincuencia Organizada Transnacional y Sus Protocolos. Así, un grupo delictivo organizado es un grupo estructurado de tres o más personas que exista durante cierto tiempo y que actúe concertadamente con el propósito de cometer uno o más delitos graves o delitos tipificados con arreglo a la convención de las Naciones Unidas (2004) con miras a obtener, directa o indirectamente, un beneficio económico u otro beneficio de orden material. 


\section{Antecedentes: deporte, apuestas y crimen}

Los juegos de azar ya sean legales o ilegales, regulados o no regulados, siempre han sido un terreno fértil para el crimen. Como Tak, Sam y Jackson (2018b: 73) destaca "donde hay deporte, hay apuestas. Y donde hay apuestas, con frecuencia, se hace trampa". Descripciones de estas prácticas pueden encontrarse tanto en la literatura científica (Ferentzy y Turner, 2009) como en el arte (el cine o la literatura, por ejemplo). ${ }^{2}$ El juego, los casinos y las apuestas representan históricamente un rasgo distintivo de la mafia y el crimen organizado (Gardiner, 1970; Haller, 1971; Reuter, 1984; Misse, 2007; Wang, 2012; Zhang y Chin, 2008).

Estos lazos han evolucionado de forma natural con las nuevas tecnologías y las posibilidades para el fraude han aumentado y se han complejizado con la evolución de los juegos de azar y la industria de apuestas deportivas. Las mayores modificaciones han sido la transición de los tradicionales mercados fuera de línea (off-line) estructurados en base a monopolios (en general públicos) a la oferta masiva y generalizada de apuestas online ofrecidas por una gran cantidad de operadores privados. Si antes los apostadores debían ir hasta una casa de apuestas para jugar a una cantidad limitada de juegos de azar, ahora pueden acceder a una gran cantidad de ofertas provenientes de distintos lugares del mundo teniendo un mero teléfono inteligente.

Las organizaciones criminales modernas continúan su vínculo tradicional con las apuestas, pero ahora la desarrollan a niveles transnacionales (UNODC-ICSS, 2016). Teniendo en cuenta que alrededor del $20 \%$ del intercambio comercial mundial proviene de actividades criminales (Dufour, 2013; Moriconi, 2018b), lo que genera un flujo de dinero negro constante, la unión de los mercados de apuestas con las nuevas tecnologías propicia una inmejorable oportunidad para el blanqueamiento de capitales y delitos transnacionales muy difícil de detectar, rastrear, investigar y, más aún, condenar (Moriconi, 2020; UNODC y IOC, 2019; Interpol y IOC, 2015; Spapens, 2017). Siguiendo la premisa básica del modelo económico del crimen de Gary Becker (1968), el mercado de apuestas deportivas actual genera incentivos para procurar altos réditos económicos a través de comportamientos desviantes, con una baja posibilidad de ser descubierto (Moriconi y De Cima, 2020a), fundamentalmente debido al anonimato y opacidad que ofrece Internet, en particular la Darknet.

Por otra parte, si bien es cierto que las apuestas se han relacionado con los deportes desde la antigüedad, también es cierto que las amenazas que el juego genera contra la deportividad y el juego limpio fueron destacadas por el padre de los Juegos Olímpicos modernos, Pierre de Coubertin, a principios del siglo XX. Para de Coubertin "el fair play" estaba "en peligro, [...] por el desarrollo de ese tipo de cáncer que se ha dejado imprudentemente arrojado a la diseminación: la locura de [...] las apuestas deportivas" (Coubertin, 1973 [1908]: 37-39).

2 Novelas como El Jugador de Dostoievski o letras de tango clásicos describen diferentes métodos de hacer trampa en las carreras de caballos, con el fin de tener éxito en las apuestas. Un ejemplo significativo es Tirate un Lance, escrito por Héctor Marcó y popularizado en la voz de Edmundo Rivero. 
El miedo expresado por de Coubertin se relaciona con los incentivos del mercado de las apuestas y su relación ética-moral con el espíritu olímpico y el juego limpio. Apuestas masivas generan masivos incentivos para manipular competiciones.

La Convención de Macolin define a la manipulación de competiciones como "un acuerdo intencional, acto u omisión destinado a alterar indebidamente el resultado o el desarrollo de una competición deportiva, con el fin de eliminar toda o parte de la naturaleza imprevisible de esa competición, con el objetivo de una ventaja indebida para sí o para un tercero" (Council of Europe, 2014: art. 3.4). La manipulación puede estar relacionada o no con las apuestas, y puede procurar beneficios deportivos o económicos.

El arreglo de partidos no relacionado con las apuestas puede estar motivado por el deseo de ganar un juego o una competición, para evitar el descenso, para obtener una ventaja competitiva en el futuro, como tener una posición privilegiada a la hora de escoger en un Draft o competir contra un equipo más débil en las siguientes rondas de un campeonato (Tak, Sam y Jackson, 2018b: 74). Al igual que en los casos de dopaje, estos ejemplos implican una "trampa para ganar" (Soebbing, Humphreys y Mason, 2013) y conseguir el éxito y/o prestigio deportivo.

Los amaños relacionados con las apuestas procuran beneficios económicos y financieros, poniendo en causa la ética deportiva y los valores olímpicos: el deseo de ganar y la búsqueda de la excelencia son puestos al margen. Se trata, en general, de hacer trampa para perder o para no rendir al máximo. El énfasis de esta práctica está en el lucro económico, no en el deporte.

El fenómeno de manipular competiciones no es una cuestión novedosa y precede a la existencia de las apuestas deportivas. Los primeros casos datan de la 98 Olimpiada de la Antigüedad. De hecho, el fraude y la corrupción son considerados como las causas principales del colapso de los Juegos Olímpicos de la Antigüedad. Aunque el amaño haya sido una práctica recurrente en la historia del deporte (Maenning, 2005), las nuevas características y volumen del mercado de apuestas generaron nuevos desafíos y problemas. El fenómeno dejó de ser una cuestión eminentemente deportiva y se transformó en una amenaza a nivel económico, social, financiero y político (Council of Europe, 2014; Carpenter, 2012). De acuerdo con Europol (2017), el crimen organizado se ve cada vez más seducido por la corrupción deportiva y esta actividad se ha convertido en una de sus principales inversiones en Europa.

La gravedad de la situación, junto con una sucesión de escándalos transnacionales, determinó que el fenómeno se convierta en un problema de política pública. En consecuencia, desde hace más de diez años se han establecidos diversos protocolos de colaboración entre entidades de diferente índole para estudiar el tema, proponer medidas y recomendaciones, establecer nuevos marcos legales y de acción y combatir el flagelo. En este sentido, el Comité Olímpico Internacional (COI) junto a la Oficina de las Naciones Unidas contra la Droga y el Delito (UNODC) han estado proponiendo marcos jurídicos para combatir el problema (IOC y UNODC, 2013; 2016) y recomendaciones para crear sistemas de denuncia protegidas (UNODC y IOC, 2019). Asimismo, el IOC estableció una asociación con la Interpol, con el objetivo de mejorar la capacidad de investigación a nivel nacional e internacional, así como proporcionar apoyo operativo al cumplimiento de los reglamentos con el fin 
de prevenir eficazmente las violaciones a la integridad deportiva (Interpol y IOC, 2015). Por su parte, la Federación Internacional de Tenis, junto con otras autoridades de gobierno del tenis, creó la Unidad de Integridad del Tenis (TIU) que posee características de policía dentro del ámbito de las competiciones oficiales y está a cargo de las sanciones por crímenes relacionados con las apuestas.

Las formas de cooperación a nivel internacional se complementan con las medidas que a nivel nacional propone la Convención del Concejo de Europa. Según la norma, cada país debe establecer una Plataforma Nacional a través de la cual todos los actores implicados en el combate a la nueva criminalidad (operadores y reguladores de apuestas, policías, poder judicial, organizaciones deportivas, asociaciones de la sociedad civil) cooperen, intercambien información y establezcan medidas de acción.

\section{Metodología y estructura}

La investigación preliminar para el desarrollo de este artículo fue realizada en el marco del proyecto Training on Protected Reporting System (T-PREG) financiado por la Comisión Europea a través de su programa Erasmus + Sport. Esta iniciativa permitió a los autores comenzar a trabajar conjuntamente y sistematizar, en base a documentos oficiales, fuentes secundarias, las áreas de riesgo presentadas a lo largo de este trabajo, que tiene, como fue expresado anteriormente, un objetivo puramente descriptivo. Las coyunturas criminales, así como la existencia de las prácticas descriptas en este texto, fueron tema de debates en diferentes focus groups y entrevistas con informantes clave ${ }^{3}$ realizados a lo largo de la implementación del proyecto T-PREG.

Las estructuras de oportunidad para el fraude o el delito son presentadas divididas en cuatro temas: (a) los nuevos incentivos para la manipulación de competiciones, (b) las estafas, como el gambling scam, y el arbitraje, (c) las debilidades de la regulación internacional y las discusiones para definir lo que son apuestas legales e ilegales, y (d) la emergencia de incentivos para crear un mercado ilegal de la derrota.

\section{El nuevo mercado de apuestas y los nuevos incentivos para la manipulación}

Los cambios más significativos en el mercado mundial de apuestas incluyen: el rápido aumento de los operadores en línea con servicios y ofertas de apuestas en todo el mundo; la transición de apuestas colectivas y/o combinadas (pool betting) a apuestas simples con probabilidades fijas (fixed-odds betting); nuevos tipos de apuestas además de la tradicional apuesta "1X2" 4 como, por ejemplo, la posibilidad de apostar en cualquier

3 Entre los entrevistados figuran operadores y reguladores de apuestas, fuentes policiales, y actores deportivos.

41 = victoria del equipo $1 ; \mathrm{X}=$ empate; 2 = victoria del equipo 2. 
evento de una competición deportiva que involucre probabilidad (spot betting); la posibilidad de colocar apuestas en directo y/o en tiempo real (in-play or live betting) en una gran cantidad de eventos deportivos; el aumento de los porcentajes de tasas de pago (payouts), que pasaron de estar debajo de $170 \%$ a estar por encima del $92 \%$ que ofrecen actualmente los operadores de apuestas online europeos licenciados; y la aparición de nuevos servicios como el mercado de apuestas cruzadas (betting exchange) o la posibilidad de cobrar por anticipado antes del final de una competencia (cash out). La tabla 1 sistematiza todos los cambios de manera comparada.

Por otra parte, hay pocos obstáculos geográficos para apostar en línea. Actualmente, los apostantes pueden hacer apuestas en todo el mundo y en tiempo real en las competiciones celebradas en cualquier lugar.

Este nuevo y complejo panorama se ha insertado en un contexto de reconocida fragilidad en lo referente a la gobernanza interna del mundo deportivo y serias deficiencias de los marcos reguladores de los nuevos mercados de juegos de azar (Moriconi y Almeida, 2019b; Kihl, Skinner y Engelberg, 2017; Tak, Sam y Jackson, 2018b; Philippou, 2019). En consecuencia, el nuevo mercado de apuestas en línea (regulado y no regulado, legal e ilegal) se ha convertido en una caja de Pandora abierta debido a los obstáculos que encuentran las autoridades judiciales, policiales y deportivas para legislar, investigar, cooperar, juzgar y sancionar, con un enfoque internacional coordinado que incluya múltiples jurisdicciones y latitudes. Estas dificultades para punir, sumadas a la posibilidad de obtener altos beneficios, ha llevado a que muchos individuos y colectivos, desde mafias transnacionales a empresarios y agentes deportivos con intereses espurios, estén procurando influir en resultados y desarrollos de competiciones o, simplemente, blanquear dinero proveniente de delitos a través de cuentas de apuestas.

Escándalos como el Calcioscommesse, ${ }^{5}$ el caso Bochum ${ }^{6}$ y el caso Hoyzer, 7 implicaron altos niveles de sofisticación tecnológica y criminalidad a escala global: redes internacionales de apostadores, un gran número de intermediarios, árbitros, dirigentes y atletas vinculados a financistas asiáticos que contratan "mulas" o "corredores". Estas mulas tenían la tarea de distribuir apuestas de sumas razonables de dinero a lo largo de diferentes corredores de apuestas de distintos países. De esta forma, los tramposos evitaban ser detectados por los sistemas de alerta y monitorización del mercado de apuestas. Un caso reciente en Portugal ha demostrado cómo los clubes de fútbol pueden ser adquiridos por las mafias extranjeras con el objetivo de blanquear dinero, crear equipos integrados por deportistas dispuestos a dedicarse a perder partidos o manipular competiciones y, de esta manera, obtener beneficios en el mercado de apuestas.

En 2011, varias figuras del fútbol, entre ellos exjugadores internacionales italianos, fueron detenidos por una presunta manipulación de los resultados.

6 En 2009, un fraude masivo fue descubierto por la Oficina de la Fiscalía de Bochum, en Alemania. De acuerdo con la investigación, al menos 200 partidos de fútbol se habían amañado en nueve países europeos a través de sobornos pagados a jugadores, entrenadores y árbitros.

7 En 2005, el árbitro de fútbol alemán Robert Hoyzer confesó su participación en amaños y apuestas en partidos de la segunda y tercera división y de la Copa de Alemania. 
Tabla 1 Características del mercado de apuestas

\begin{tabular}{|c|c|c|}
\hline & \multicolumn{2}{|c|}{ Cambios } \\
\hline & Características antes de la era de Internet & Características del mercado de apuestas actual \\
\hline Territorio & $\begin{array}{l}\text { Con muy pocas excepciones, no era posible } \\
\text { realizar apuestas fuera de la jurisdicción del } \\
\text { consumidor. }\end{array}$ & $\begin{array}{l}\text { Es posible realizar apuestas en todo el mundo y } \\
\text { utilizar operadores legales, ilegales, con } \\
\text { licencia, sin licencia o irregulares. En los países } \\
\text { que restringen a los operadores ilegales, es } \\
\text { posible, no obstante, acceder a ellos usando ID } \\
\text { falsos o robados y abriendo cuestas bancarias } \\
\text { en paraísos fiscales u offshore. En los países } \\
\text { que no tienen políticas contra los operadores } \\
\text { ilegales y se puede acceder a cualquier } \\
\text { operador, simplemente se necesita abrir una } \\
\text { cuenta donde el apostador desea, sea en un } \\
\text { operador nacional o en uno extranjero. }\end{array}$ \\
\hline $\begin{array}{l}\text { Número de } \\
\text { operadores }\end{array}$ & $\begin{array}{l}\text { En la mayoría de los países, las apuestas } \\
\text { deportivas se prohibieron o fueron } \\
\text { proporcionadas por un solo operador } \\
\text { (monopolio). Había alrededor de } 300 \\
\text { operadores de apuestas deportivas } \\
\text { autorizados (caballos y galgos excluidos) en } \\
\text { todo el mundo. En algunos países, las redes } \\
\text { ilegales eran fuertes. La mayoría de los } \\
\text { operadores con licencia estaban localizados } \\
\text { en el Reino Unido, que daba licencias } \\
\text { individuales a los operadores de apuestas. }\end{array}$ & $\begin{array}{l}\text { Más de } 60 \text { jurisdicciones alrededor del mundo } \\
\text { otorgan alguna forma de licencia para los } \\
\text { operadores de apuestas (online o tiendas). El } \\
\text { número de operadores de apuestas con licencia } \\
\text { varía de } 3000 \text { a } 10.000 \text {. Además, hay un gran } \\
\text { número de corredores de apuestas (físicos u } \\
\text { online) totalmente ilegales que ni siquiera están } \\
\text { registrados (por ejemplo, en diferentes países } \\
\text { de Asia, en Costa Rica, en Italia, en UE, etc.). }\end{array}$ \\
\hline \multirow{4}{*}{$\begin{array}{l}\text { Productos } \\
\text { principales } \\
\text { ofrecidos }\end{array}$} & \multirow{4}{*}{$\begin{array}{l}\text { Apuestas colectivas y/o combinadas (pool } \\
\text { betting: totalisator o parimutuel) sin riesgo } \\
\text { para el operador de apuestas. En las } \\
\text { apuestas combinadas o colectivas, el } \\
\text { consumidor (apostador) no sabe de } \\
\text { antemano cuánto puede ganar. El dinero } \\
\text { recaudado de las apuestas realizadas es } \\
\text { colocado en un pozo y el porcentaje del pozo } \\
\text { destinado a premios se reparte de manera } \\
\text { equitativa entre los ganadores. Si hay } \\
\text { muchos ganadores, se reducen las } \\
\text { ganancias. Algunos ejemplos son Totobola, } \\
\text { Quiniela o Euromillones. } \\
\text { En los noventa, este tipo de apuestas } \\
\text { representaba más del } 90 \% \text { del mercado } \\
\text { mundial de apuestas deportivas legales. Hoy } \\
\text { representa menos del } 10 \% \text {. } \\
\text { En Italia, la facturación del juego de apuestas } \\
\text { deportivo más famoso, el Totocalcio, cayó } 3 \\
\text { mil millones de euros en la década de } 1990 \text { a } \\
150 \text { millones en la actualidad. }\end{array}$} & $\begin{array}{l}\text { Apuestas de probabilidades fijas previamente } \\
\text { calculadas por el operador (fixed-odds betting), } \\
\text { que acepta un riesgo financiero. En este tipo de } \\
\text { apuestas fijas, el apostador puede calcular sus } \\
\text { potenciales ganancias por adelantado. Las } \\
\text { ganancias resultan de multiplicar la probabilidad } \\
(\text { odd }) \times \text { la cuantía de la apuesta } \\
(\text { ganancias = apuesta } \times \text { odds). }\end{array}$ \\
\hline & & $\begin{array}{l}\text { Por ejemplo, un apostador puede apostar a que } \\
\text { Roger Federer ganará su partido contra Rafael } \\
\text { Nadal. Si la cuota que el operador ofrece por el } \\
\text { triunfo de Federer es } 1,40 \text {, un apostador que } \\
\text { apuesta } 100 \text { euros ganará } 100 \times 1,4 \text { euros si el } \\
\text { tenista suizo gana. }\end{array}$ \\
\hline & & $\begin{array}{l}\text { En los noventa, las apuestas de probabilidades } \\
\text { fijas representaban menos del } 10 \% \text { de las } \\
\text { apuestas deportivas legales en todo el mundo. }\end{array}$ \\
\hline & & $\begin{array}{l}\text { En la actualidad, significan más del } 90 \% \text { del } \\
\text { mercado. }\end{array}$ \\
\hline
\end{tabular}




\begin{tabular}{|c|c|c|}
\hline $\begin{array}{l}\text { Tipos de } \\
\text { apuestas }\end{array}$ & $\begin{array}{l}\text { En la década de 1990, había un tipo de } \\
\text { apuesta deportiva "estrella": } 1 \text { X2 (apostar por } \\
\text { el triunfo local, el empate o el triunfo } \\
\text { visitante). } \\
\text { En algunos países, era posible apostar con } \\
\text { otros tipos de apuestas (sobre todo en los } \\
\text { pocos países que ofrecian apuestas de } \\
\text { probabilidades fijas), pero el mercado para } \\
\text { estos productos era muy bajo: apuestas } \\
\text { sobre resultados exactos, apuestas con } \\
\text { hándicap (handicap betting), spread, etc. } \\
\text { El mercado ilegal, incluso en aquellos años } \\
\text { solía ofrecer la mayor parte de los productos. }\end{array}$ & $\begin{array}{l}\text { En la actualidad, } 1 X 2 \text { sigue siendo el tipo de } \\
\text { apuestas líder en la mayoria de los países. Sin } \\
\text { embargo, dos factores han cambiado por } \\
\text { completo la estructura del mercado: } \\
\text { a. Algunos otros tipos de apuestas tienen en la } \\
\text { actualidad una cuota importante de mercado } \\
\text { para los operadores, especialmente en línea. } \\
\text { Entre esos tipos de apuestas con cuotas de } \\
\text { mercado importante se encuentran handicap } \\
\text { betting, número total de goles (o puntos, o sets } \\
\text { o juegos) o apuestas sobre el resultado exacto } \\
\text { de un evento, etc. } \\
\text { b. Gracias a las posibilidades que ofrece el } \\
\text { mercado online y las nuevas tecnologías, } \\
\text { sumado a que diversas compañías venden } \\
\text { estadísticas en tiempo real sobre muchos } \\
\text { aspectos de las competiciones (como el número } \\
\text { de tarjetas, números de tiros de esquina o } \\
\text { laterales, datos individuales sobre desempeño } \\
\text { de los jugadores, etc.), han aparecido muchos } \\
\text { nuevos tipos de apuestas en vivo sobre } \\
\text { particularidades del juego o cuestiones } \\
\text { puntuales a ocurrir en el desarrollo del evento. }\end{array}$ \\
\hline $\begin{array}{l}\text { Momento para } \\
\text { colocar las } \\
\text { apuestas }\end{array}$ & $\begin{array}{l}\text { Antes de } 2000 \text {, las apuestas debian ser } \\
\text { colocadas antes del inicio de las } \\
\text { competiciones: los operadores cerraban las } \\
\text { apuestas justo antes del saque inicial o la } \\
\text { largada. Por esta razón, la variedad de tipos } \\
\text { de apuestas ofrecidas era mucho menos } \\
\text { significativo. }\end{array}$ & $\begin{array}{l}\text { Alrededor de } 2000 \text {, algunos operadores } \\
\text { comenzaron a ofrecer apuestas en vivo y/o } \\
\text { durante los eventos. De esta manera, se hizo } \\
\text { posible colocar apuestas después del inicio de } \\
\text { las competiciones. Para este tipo de apuestas, } \\
\text { las tasas de pago y probabilidades varian } \\
\text { dependiendo del desarrollo del evento. } \\
\text { Por ejemplo, si un equipo es favorito y tiene una } \\
\text { tasa de pago de } 1,20 \text { y transcurridos } 30 \\
\text { minutos va ganando } 2 \text { a } 0 \text {, las probabilidades de } \\
\text { que ese equipo gane son aún mayores que al } \\
\text { inicio, por lo que la tasa de pago baja (por } \\
\text { ejemplo, a } 1,05 \text { ). En pocas palabras, los } \\
\text { operadores van ofreciendo tasas de pagos más } \\
\text { bajas a medida que las probabilidades de que } \\
\text { algo suceda son mayores, y más altas cuando } \\
\text { las probabilidades de que algo acontezca son } \\
\text { menores. }\end{array}$ \\
\hline Deportes & $\begin{array}{l}\text { En la década de los noventa, más del } 98 \% \\
\text { de las apuestas deportivas legales eran } \\
\text { sobre fútbol. } \\
\text { En los mercados ilegales, por ejemplo, los } \\
\text { desarrollados en los Estados Unidos, } \\
\text { también había una buena cantidad de } \\
\text { apuestas en fútbol americano, béisbol, y } \\
\text { baloncesto, mientras que en los } \\
\text { desarrollados en la India se apostaba mucho } \\
\text { en cricket. }\end{array}$ & $\begin{array}{l}\text { Hoy en día, los operadores de apuestas ofrecen } \\
\text { apuestas en más de } 50 \text { deportes diferentes. El } \\
\text { fútbol, en la actualidad, representa menos del } \\
65 \% \text { del mercado. }\end{array}$ \\
\hline
\end{tabular}




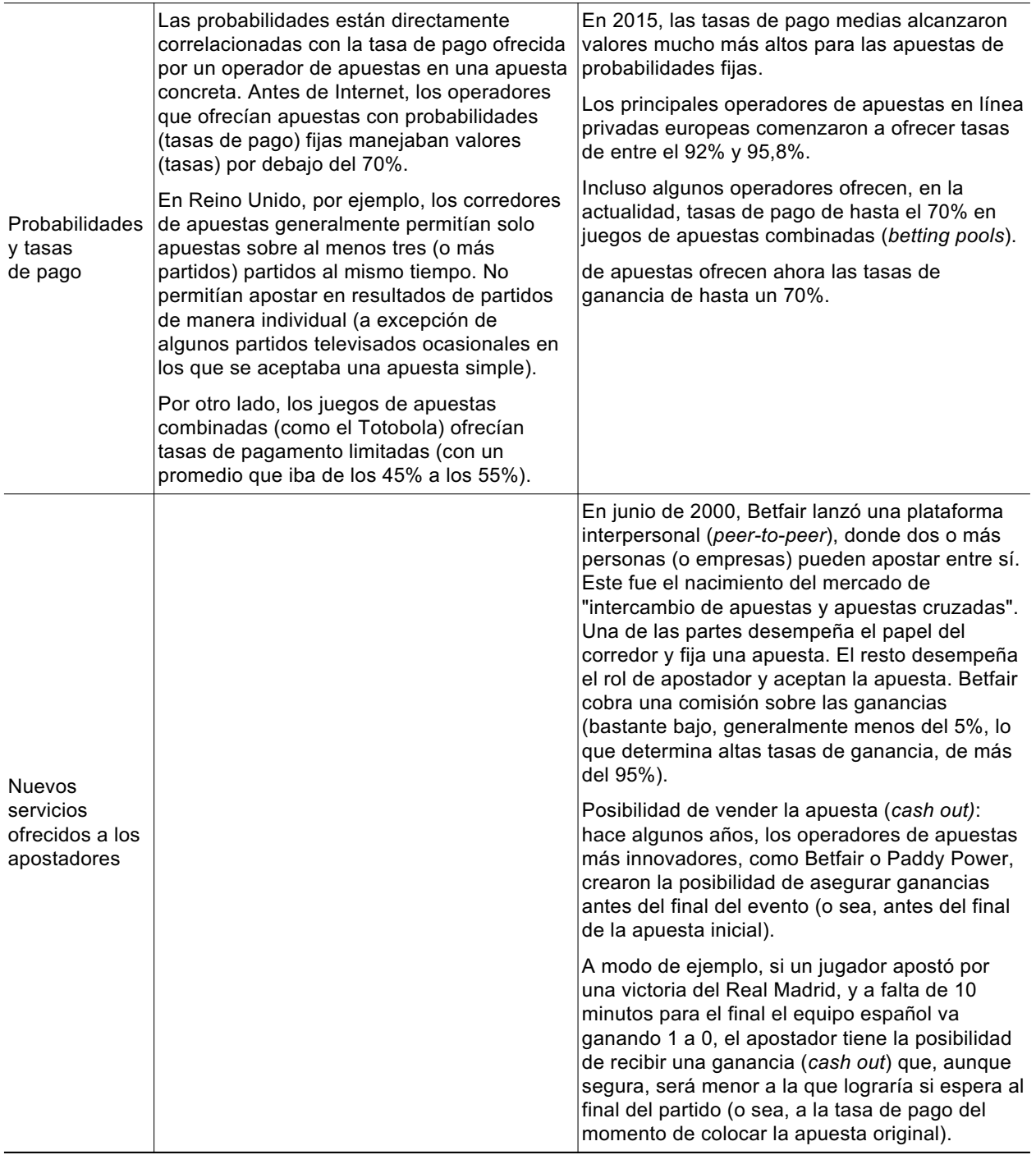

Fuente: IRIS (2017: 28-29); La Sorbonne / ICSS (2014); Moriconi y Almeida (2019b). 
Por tener menor número de participantes, los deportes individuales, como el tenis o el boxeo, serían más fácil de ser manipulados que os deportes colectivos. Por otro lado, algunos autores sostienen que los jugadores que acaban de empezar sus carreras y necesitan recursos para continuar desarrollándolas (como los jugadores de tenis que participan en los torneos Futures y Challenger), junto a aquellos deportistas que están al final de sus carreras y no se han garantizado una estabilidad económica, son los principales blancos de los manipuladores profesionales (Hill, 2008; Moriconi, 2018b; 2020). Los árbitros también son objetivos de presión constante y se perciben como un colectivo con múltiples áreas de riesgo y focos de corruptibilidad (Moriconi y Teixeira-Diniz, 2016; Moriconi, 2020).

Entre los factores que pueden llevar a un deportista a participar en la manipulación de competencias figuran el retraso en el pago de salarios (FIFPro, 2012; Hill, 2010; Moriconi y De Cima, 2020b); los problemas económicos individuales; las adicciones; la avaricia y la desintegración de valores éticos; el interés y la presión de los agentes deportivos, intermediarios y patrocinadores; la falta de control interna sobre los flujos de dinero, y la falta de transparencia y de altos estándares de gobernanza en la gestión de clubes, federaciones y órganos rectores del deporte (Tak, Sam y Jackson, 2018a; TIAC, 2014, 2018; Han, 2020).

La suma de todos estos factores ha llevado a que el fenómeno del amaño de competiciones sea considerado como la mayor amenaza para la integridad del deporte internacional (Carpenter, 2012; Gokhale, 2009). Según el ex presidente del Comité Olímpico Internacional (COI), Jacques Rogge, el dopaje afecta a un atleta individual, pero el impacto de la manipulación de resultados es mucho más grande porque afecta a toda la competición. ${ }^{8}$

Sin embargo, los incentivos para que la delincuencia organizada se infiltre en el mundo de las apuestas deportivas va mucho más allá de las históricas manipulaciones y amaños e, incluso, no se limita a cuestiones ilegales o criminales. La complejidad del nuevo mercado de apuestas online global, junto con las nuevas tecnologías, crean nichos que fomentan nuevos fraudes que nada tienen que ver con la influencia externa sobre el desarrollo de los eventos deportivos.

\section{Trampa y fraudes sin necesidad de manipular}

En el nuevo mundo de las apuestas online, cada deporte genera sus propios nichos para el fraude. En el caso de los deportes de raqueta, o incluso de voleibol, ha surgido una nueva forma de engañar a las casas de apuestas. En estos deportes, los puntos se producen uno tras otro, y los operadores de apuestas ofrecen apuestas en directo sobre quién será el ganador del punto siguiente. Si hubiese un retraso entre el final de un punto y la transmisión del resultado al operador de apuestas, un apostador que se encontrara en el estadio, y tuviese un teléfono inteligente conectado a Internet, podría aprovechar el lapso de tiempo para apostar en el ganador de

Ver: http://www.fairplayinternational.org/sports-biggest-threats-corruption-and-matchfixing 
los puntos después de saber, justamente, quién ha sido el ganador. Esta estafa, conocida como gambling scam, ha estado sucediendo en diversos torneos: ${ }^{9}$ los jueces son sobornados para retrasar la entrada de puntos en el sistema de puntuación oficial con el fin de permitir que grupos de apostadores profesionales (que están presentes en el estadio o que desde el estadio envían información rápida a los apostadores) realicen apuestas seguras y cosechen beneficios.

Por otro lado, es un mundo con un elevado flujo de dinero negro, ${ }^{10} \mathrm{el} \mathrm{mercado}$ de apuestas deportivas en línea se convierte en un medio de lavado de dinero, incluso sin necesidad de manipular resultados. El propio sistema genera situaciones en las que es posible apostar dinero con bajo riesgo y sin la necesidad de participar en el amaño de partidos o sobornar a los agentes externos.

Por ello, diversos apostadores profesionales han comenzado a utilizar las apuestas deportivas online atraídos por las oportunidades para realizar arbitraje y obtener altas tasas de ganancia. El arbitraje consiste en un análisis global del mercado en procura de encontrar ofertas de tasas de pago diferentes en distintas casas de apuestas que, mezcladas, produzcan la posibilidad de lograr apuestas seguras. En este marco, aplicando técnicas de inversión financiera, se pueden obtener ganancias sin necesidad de corromper a alguien.

A diferencia del amaño de partidos, donde un manipulador tiene que "comprar" a un actor deportivo (atleta, jugador, entrenador, árbitro) los apostadores que practican el arbitraje pueden operar legalmente en los márgenes del sistema y conseguir tasas de retorno del $100 \%$ al $102 \%$, o sea, conseguir lo que el mundo de los juegos azar se conoce como apuesta segura.

El modus operandi es abrir cuentas en una gran cantidad de operadores a nivel mundial establecidos en distintas jurisdicciones. En algunas jurisdicciones, que funcionan como paraísos fiscales, el trabajo es bastante simple: el apostador puede abrir cuentas en los diez o quince operadores registrados que mejores probabilidades ofrezcan, sabiendo que su identidad, edad o cuenta bancaria raramente serán verificados. Luego, mediante la utilización de softwares, algoritmos y hardware que mejoran la potencia de cálculo, la velocidad de acceso al mercado y la frecuencia de transacción de apuestas se procuran las mejores ofertas de mercado en diferentes eventos y se calculan las tasas de retorno que se pueden conseguir.

De esta manera, el nombre del apostador profesional se disuelve en el ciberespacio y pasa desapercibido por los controles de los operadores, reguladores y sistemas de monitorización. Debido a la rentabilidad asegurada, los apostadores profesionales (individuales o colectivos) son procurados por sindicatos del crimen o evasores, quienes se convierten en financistas con el objetivo de blanquear sus capitales.

9 En esta nota del periódico The Guardian, Sean Ingle presenta algunos casos ocurridos en el mundo del tenis: https://www.theguardian.com/sport/2016/feb/09/revealed-tennis-umpires-secretly-banned-gambling-scam

10 Debido al gran flujo de dinero negro en todo el mundo negro, diversas instituciones internacionales, como el Banco Mundial y los gobiernos de los diferentes países han recomendado y/o promovido procesos de amnistía tributaria. Para ampliar los efectos éticos de la naturalización de las amnistías fiscales sobre la legitimidad de la racionalidad jurídica ver Moriconi (2018b). 
Estas nuevas prácticas, que incluyen a grupos de apostadores profesionales, jugadores que realizan arbitraje y otros que utilizan algoritmos, plantea serios desafíos a los operadores tradicionales. Básicamente, lo que los jugadores profesionales procuran es vencer al operador y a al mercado.

A continuación, mostramos un ejemplo clásico de una apuesta segura para lavar dinero en un partido de fútbol de la Liga de Campeones de la UEFA citado por Kalb y Verschuuren (2013: 56). El partido fue jugado por el AC Milán contra el FC Barcelona, el 28 de marzo de 2012. Para fórmulas 1X2, existieron, en diferentes operadores de apuesta, las siguientes probabilidades disponibles en el mercado:

- 5,25 para una victoria del AC Milán,

- 4 por un empate,

- $\quad$ 1,73 para una victoria del FC Barcelona.

Con esta oferta de pagos, un apostador podía blanquear capital a muy poco coste apostando en las tres hipótesis, es decir:

- Apostar en 1, o sea en la victoria del Milán del operador A

- Apostar en X, en el empate en el operador B

- Apostar en 2, en la victoria del Barcelona en el operador C

Apostando 100 euros, por ejemplo, el apostador se garantizaba un retorno de 98,18 euros independientemente del resultado del partido (ver tabla 2):

- $\quad$ el porcentaje que se apostó en " 1 " es $1 /(1+5,25 / 1,73+5,25 / 4)=18,7 \%$ $(18,7$ euros)

- $\quad$ el porcentaje que se apostó en " $X$ " es $1 /(1+4 / 1,73+4 / 5,25)=24,55 \%$ (24,55 euros)

- $\quad$ el porcentaje que se apostó en " 2 " es $1 /(1+1,73 / 4+1,73 / 5,25)=56,75 \%$ (56,75 euros)

Por lo tanto, independientemente de cómo termine el partido, si el apostador fuese un criminal que busca lavar dinero, con esta apuesta tendría la posibilidad de justificar 98,2 euros en ganancias. Es decir, estaría blanqueando 98,20 de los 100 euros en negro, lo que implica una mínima pérdida de $1,8 \%$ mucho menor incluso que cualquier tasa de amnistía fiscal impulsada en algún estado europeo y, lógicamente, muchísimo menor a cualquier tasa impositiva legal.

Si bien en este caso se trata de un retorno, el arbitraje permite encontrar tasas de retorno que superan el $100 \%$, es decir, que implica que la apuesta colocada sea totalmente segura. En el ejemplo anterior, esto hubiese sucedido si, simplemente, se encontraba en el mercado una oferta ligeramente superior (por ejemplo 1,79) para el triunfo del Barcelona. En ese caso, el apostador podría apostar 100 euros al triunfo del Milán, 131,25 al empate y 293,30 euros al triunfo del Barcelona (ver tabla 3). Con una inversión de 524,55 euros, el apostador se asegura un retorno de 525. 


\begin{tabular}{lcccc}
\hline AC Milan - FC Barcelona & 1 & $X$ & 2 & Total \\
\hline Probabilidades (odds) & 5,25 & 4 & 1,73 & \\
Operador seleccionado & A & B & C & 100,00 \\
Cantidad apostada (em euros) & 18,70 & 24,55 & 56,75 & 98,20 \\
Ganancias si gana el AC Milán (1)(em euros) & 0 & 0 & 98,20 & 98,20 \\
Ganancias si hay un empate (X) (em euros) & 0 & 98,20 & 0 & 98,20 \\
Ganancias si gana el FC Barcelona (2) (em euros) & 98,20 & 0 & 0 & \\
\hline
\end{tabular}

Fuente: Kalb y Verschuuren (2013).

Ejemplo de apuesta segura (tres posibilidades)

\begin{tabular}{lcccc}
\hline & 1 & $X$ & 2 & Total \\
\hline Probabilidades (odds) & 5,25 & 4 & 1,79 & \\
Cantidad apostada (em euros) & 100,00 & 131,25 & 293,30 & 524,55 \\
Ganancias si gana (1) (em euros) & 525,00 & 0 & 0 & 525,00 \\
Ganancias si hay un empate (X) (em euros) & 0 & 525,00 & 0 & 525,00 \\
Ganancias si gana (2) (em euros) & 0 & 0 & 525,00 & 525,00 \\
\hline
\end{tabular}

Fuente: información propia.

Ante la gran cantidad de operadores a nivel mundial, los algoritmos de los apostadores profesionales suelen encontrar este tipo de jugadas con ganancia garantizada. Para encontrar una apuesta segura, los dispositivos y programas de monitorización de los apostadores profesionales comparan las mejores cuotas ofrecidas en el mercado global. Este proceso consiste en calcular la probabilidad de las tasas de pago ofrecidas (100 divido por la tasa de pago) y sumarlas, procurando sumas que no lleguen al $100 \%$.

Anteriormente fue presentado un ejemplo para un caso de tres posibilidades (1X2). Veamos ahora un ejemplo de dos posibilidades, como puede ser un partido de tenis, de básquet, un deporte de combate. En un partido de tenis se enfrentan Roger Federer y Rafael Nadal. En España se espera que Nadal sea favorito, en Suiza hay preferencia por Federer. Estas diferencias se expresan en el mercado, dado que el partido es muy parejo. La monitorización arroja que la mejor oferta para el triunfo de Federer es 2,10 y para Nadal 2,00. Las probabilidades por tanto serían 47,6\% para la victoria de Federer (100 dividido por 2,10$)$ y $50 \%$ para Nadal (100 dividido por 2). La suma de las dos probabilidades es de $97,6 \%$, por tanto, siendo debajo de $100 \%$, estamos ante una apuesta segura.

El apostador juega 100 euros en la victoria de Federer y 105 en la victoria de Nadal (ver tabla 4). Habiendo hecho una inversión de 205 euros, siempre logrará ganar 210 euros, fuera cual fuera el resultado. Si en vez de cantidades de tres cifras, un operador tiene muchas cuentas abiertas y puede apostar millones de dólares, las ganancias seguras son muy elevadas. 


\begin{tabular}{lccc}
\hline & 1 & 2 & Total \\
\hline Probabilidades (odds) & 2,10 & 2,00 & \\
Cantidad apostada (em euros) & 100,00 & 105,00 & 205,00 \\
Ganancias si gana Federer (1) (em euros) & 210,00 & 0 & 210,00 \\
Ganancias si gana Nadal (2) (em euros) & 0 & 210,00 & 210,00 \\
\hline
\end{tabular}

Fuente: información propia.

\section{Apuestas legales e ilegales: un dilema de política pública}

Otro de los factores conflictivos del nuevo mercado mundial de apuestas es el debate sobre la forma de establecer la diferencia entre las apuestas legales e ilegales. La falta de una definición consensuada del concepto de apuesta ilegal ha sido, y continúa a ser, una de las principales controversias políticas que rodean a la implementación de la Convención de Macolin. Mientras algunos sectores creen que la legalidad de las apuestas debe ser resuelta de acuerdo con la jurisdicción de la licencia del operador, otros sectores sostienen que se debe definir de acuerdo a la jurisdicción del consumidor.

Más allá de este debate, y debido a que las sumas de dinero cada vez más grandes que mueve el mercado de apuestas deportivas, hay pocas dudas sobre la atracción del crimen organizado por este nuevo sector (Hill, 2008). Aunque debido a la extensión y complejidad del merado es imposible saber con exactitud cuánto dinero se mueve, las estimaciones menores indican que serían alrededor de 300 billones de dólares, mientras que las mayores estiman que se mueven alrededor de 1500 billones de dólares. En este marco, si la condición para distinguir entre apuestas en mercados legales o en mercados ilegales fuese la tenencia de una autorización en la jurisdicción del consumidor, entonces, en las actuales condiciones, los mercados ilegales representarían el 82\% del mercado global (ver tabla 5). En consecuencia, teniendo en cuenta esos datos, se advierte que la cantidad de dinero que circula en los mercados de apuestas sin ningún (o con poco) control es enorme.

Por otra parte, el promedio de retorno en el mercado de apuestas ilegal, es decir el porcentaje del dinero invertido que luego se destina a premios, es muy alto, rondando el $96,2 \%$. Esto se debe a los pocos controles antes explicados, que implican también menos o ningún impuesto y, por tanto, la posibilidad de que los operadores logren buenas ganancias ofreciendo un producto mucho más interesante al apostador, que procura mayores posibilidades de ganar. Esto acarrea dos problemas. En primer lugar, siendo que la mejor tasa de retorno se materializa en el mercado con mejores pagos por apuestas (odds), los apostadores que puedan optar por un operador ilegal y uno legal podrán optar por el ilegal y ganar más con el mismo riesgo. En términos simples e hipotéticos, si un apostador tiene decidido apostar 100 euros en que el equipo A va a ganar, podrá optar por un operador legal y ganar 150 euros o colocar la apuesta e un operador ilegal que le pague 180. El riesgo y el objetivo de la apuesta son los mismo: el equipo A debe ganar. Pero el premio es más 


\begin{tabular}{lccc}
\hline & Mercado legal & Mercado ilegal & Total \\
\hline Cantidades apostadas & $85.000(18 \%)$ & $390.000(82 \%)$ & $475.000(100 \%)$ \\
(ventas) - millones de euros & $82,4 \%$ & $96,2 \%$ & $93,7 \%$ \\
Tasa de retorno $(\%)$ & $15.000(50 \%)$ & $15.000(50 \%)$ & $30.000(50 \%)$ \\
GGY - millones de euros & &
\end{tabular}

Fuente: IRIS (2017: 40).

alto en el operador ilegal. Dicho esto, también es necesario explicar que lo "ilegal" en este caso no implica altos riesgos para el apostador, y esto se ve claramente en el conflicto que ha abierto la Convención de Macolin en Europa por causa de las críticas que Malta mantiene con el Concejo de Europa y que aún se mantienen debido a un litigio legal. Para Malta, cualquier operador radicado en su territorio (donde los impuestos para el juego son muy bajos), tiene el derecho de comercializar libremente en toda la Unión Europea. Para otros países, como por ejemplo Portugal, si ese operador no toma una licencia nacional y paga sus impuestos en el país, será un operador considerado ilegal. En la práctica lo que acontece es que los apostadores continúan a acceder mediante Internet a operadores tanto legales como ilegales, y ambos tipos de operadores aceptan la abertura de nuevas cuentas y cumplen con los pagos. En resumen, en muchos casos, los apostadores son libres de escoger entre un operador legal y uno ilegal.

En segundo lugar, la alta tasa de retorno del mercado ilegal, junto a los pocos controles, determina que sea mucho más apetecible para aquellos jugadores que procuren lavar dinero. De acuerdo con Sandywell (2010:45) los operadores de apuestas, los empleados, los clientes y las terceras partes no deseadas se misturan anónimamente, creando estructuras de oportunidad para múltiples formas de delitos y fraudes y para construir relaciones rizomáticas entre criminales y víctimas".

\section{La emergencia del mercado (ilegal) de la derrota}

El nuevo mercado online de apuestas deportivas también modifica las bases éticas de la actividad deportiva profesional. Mientras que, en la lógica deportiva tradicional, un partido o un juego es parte de un objetivo a mayor a mediano o largo plazo, como acceder a un play-off, a una final, o ganar un campeonato; en el nuevo mercado de apuestas cada punto en juego, cada partido, cada competencia y cada evento deportivo es un fin en sí mismo.

El problema es que este juego o evento (fin en sí mismo) blanco de apuestas se construye sobre el trabajo de un ser humano o un grupo de seres humanos (deportistas) que desempeñan tareas y misiones. Al ser transformados en objetos de apuesta, esos actores deportivos son el blanco de expectativas éticas y económicas externas.

Para conseguir el objetivo final de salir campeón o ganar una competición, no siempre la mejor estrategia es desempeñarse al cien por ciento de las capacidades 
individuales. Sin embargo, las nuevas apuestas sobre factores intermedios o colaterales de un evento deportivo, como las tarjetas amarillas, los puntos parciales, la cantidad de faltas o las series de clasificación, modifican la lógica del triunfo final, imponiendo imperativos éticos sobre desempeñarse de la mejor manera posible a lo largo de todo el evento deportivo blanco de apuesta.

Para un jugador de tenis, por ejemplo, no es necesario jugar cada juego (game), o incluso cada set, como si fuese el último, el definitorio. El o la tenista puede decidir ahorrar energía en uno de los sets, perderlo, y ganar los demás. Perdiendo un set y ganando el resto, siempre ganará el partido, o sea, logrará el objetivo deportivo primordial. Este ejemplo, como muchos otros, enfatizan la actual delgada línea establecida entre la estrategia deportiva y la integridad o falta de ética. De hecho, en los deportes de raqueta, esta paradoja ha llevado a que algunos amañadores profesionales ya no procuren sobornar a jugadores para que pierdan sus partidos, sino para que pierdan un set frente a rivales más débiles, o pierdan una cantidad de juegos establecidas en el set. Todo ello sin necesidad de que el deportista pierda su partido y ponga en juego su carrera. Es una relación "win-win".

Los imperativos éticos del mercado de apuestas online son diferentes a la lógica deportiva tradicional, pues las expectativas de las apuestas en vivo son que los deportistas y/o atletas se desempeñen al máximo de sus posibilidades a lo largo de todo el desarrollo de un partido o una competencia. De hecho, el cálculo de las probabilidades (odds) y las ofertas de apuestas siguen ese patrón ético.

Este imperativo ético, paradójicamente, crea un dilema ético en relación con las prohibiciones de apostar impuestas a deportistas y atletas (Moriconi y De Cima, 2020a). Los órganos de gobierno del deporte, las federaciones, las ligas, e incluso algunos códigos penales (como el portugués) establecen que los atletas y otros agentes del mundo del deporte no pueden apostar. En algunos casos, la prohibición se limita a las competiciones en las cuales participan, en otros al deporte en el que compiten, y en los casos más extremos se prohíben las apuestas en cualquier evento deportivo. La decisión se basa en la necesidad de limitar las posibilidades de manipulación y amaño y promocionar la integridad entre los atletas. Esta medida disciplinaria, además, ayudaría a proteger la imprevisibilidad de los resultados de las competiciones.

Pero la paradoja va más allá: (a) a los jugadores y atletas se los convierte (muchas veces sin su consentimiento o aprobación) en los motores del multimillonario mercado de apuestas; (b) es necesario exigirles a ellos ética e integridad para que el mercado de apuestas sobre su trabajo (como deportistas) sea legítimo, transparente y sostenible; (c) surgen una serie de iniciativas de negocio que, a través de ese mercado que transforma a los deportistas en objetos blanco de apuestas, una multitud de personas pueden ganar dinero gracias al resultados del trabajo que esos seres humanos (deportistas) realizan; (d) pero a esos seres humanos (que con su trabajo hacen posible la existencia del mercado de apuestas en el que muchas personas ganan dinero) se les niega la posibilidad de ganar dinero extra por cumplir con la obligación que a ellos se les exige, que es desempeñar su tarea "de la mejor manera posible" (perform to their best) para ganar sus competiciones. 
En síntesis, la paradoja ética no se relaciona con el hecho de prohibir a los deportistas de apostar en sus propias competiciones, sino en la prohibición de que los agentes deportivos (que hacen posible la existencia del mercado de apuestas) no tengan permiso para apostar en su triunfo o en el triunfo de su equipo, que es, de hecho, lo que las normas de integridad de los mercados de apuestas les demanda. Por supuesto, no sería ético, y sería potencialmente peligroso, que un equipo o un jugador apostara en contra de su deber de ganar, pero nunca sería un peligro apostar por su triunfo o, en otras palabras, por desempeñarse de la mejor manera posible para lograr un resultado positivo, que es el imperativo ético que el mercado de apuestas demanda.

En este sentido, Hill (2019) cuestiona que la industria de la integridad deportiva, a partir de enfatizar la necesidad de implementar campañas de educación y prevención, creó un paradigma en el que los culpables son los atletas. El fenómeno es presentado como una falla moral de los atletas (Tak, Sam y Jackson, 2018a) que carecen de información y concientización. Se parte de la premisa de que los actores deportivos deben ser éticamente educados por supuestos ejecutivos honestos contratados por las organizaciones deportivas (Hill, 2019). Justamente, son estos órganos instituciones deportivos, las ligas, los clubes, las federaciones quienes lucran directamente con el mercado de apuestas, recibiendo publicidad o financiamiento.

Esta incoherencia ética, de hecho, genera graves obstáculos a la implementación de las políticas públicas relacionados con la integridad del deporte y el mercado de apuestas. Diversos proyectos europeos ${ }^{11}$ han demostrado que, a pesar de estas normativas, son muchos los atletas que continúan a apostar, y muchos de ellos lo hacen en sus propias competiciones e incluso en sus propios partidos. Las apuestas, que tradicionalmente son una extendida práctica cultural al interior del deporte, son utilizadas como motivación individual y colectiva (Moriconi y De Cima, 2019b). Pero, además, los atletas se quejan de la forma como son creadas las políticas públicas, en una perspectiva de arriba abajo sin la participación u opinión de los actores que van a ser las personas atingidas por esas políticas. Obviamente, los atletas también reconocen que la amplitud del mercado de apuestas, que alcanza a competiciones o deportistas que cobran salarios ridículos comparados con las ganancias que se pueden conseguir apostando o que directamente no cobran (por tener sus salarios en atrasos), abre un "mercado ilegal" alrededor de la derrota (Moriconi, 2020; Tak, Sam y Jackson, 2018a).

Si los valores olímpicos del deporte tradicional giran en torno a un mercado legal en el que el triunfo y el buen desempeño son los indicadores de éxito y prestigio. Este éxito, en una sociedad de consumo (Sandel, 2012), obviamente se materializa en ganancias económicas. Con el nuevo mercado de apuestas, sin embargo, los que aparece es un rentable mercado alrededor de la derrota, sobre todo para todos aquellos atletas que no tengan el talento ni la dedicación suficiente para llegar a la cima. En esos casos, un deportista puede optar por desestimar los éxitos deportivos

11 Ver por ejemplo los reportes finales de los proyectos AMATT (https://www.amatt.eu/) y T-PREG (http://www.tpreg-training.eu/), financiados por la Comisión Europea a través del programa Erasmus + Sport. 
y centrarse en la manipulación de sus juegos en procura de amasar fortuna alrededor del mercado de apuestas.

\section{Conclusiones}

Este artículo se dedicó a describir cómo las nuevas posibilidades tecnológicas han transformado radicalmente el mercado de apuestas deportivas, generando estructuras de oportunidad para prácticas lícitas e ilícitas. Dadas las características de este nuevo mercado, los sindicatos del crimen y los apostadores profesionales se han infiltrado en el mundo del deporte para lucrar con las apuestas. A pesar de que esta situación ha transformado a la manipulación de competiciones en un problema político que transciende el mundo del deporte y, como explica la Convención de Macolin, tiene aristas financieras, criminales, judiciales y sociales, este trabajo demuestra que los fraudes y las trampas en el nuevo mercado de apuestas van mucho más allá del amaño.

Existen diversas prácticas, algunas incluso legales, que permiten que apostadores profesionales defrauden al mercado sin necesidad de tener que influir en el desempeño de los actores deportivos. La magnitud del mercado y de ofertas de tasas de pago permiten la posibilidad de arbitrarlo en busca de apuestas seguras que, con cierta frecuencia, permiten el blanqueamiento de capitales. Teniendo en cuenta la rentabilidad de diversas actividades ilegales y el flujo de dinero negro en el mundo actual, el mercado de apuestas online global genera múltiples incentivos para que los sindicatos del crimen se acerquen a él.

Por si fuera poco, las posibilidades de fraudes y delitos se crean en medio del marco opaco de la red, creando diversos obstáculos jurisdiccionales, técnicos y económicos para la prevención, detección, investigación y sanción de las actividades ilegales, y generando áreas de oportunidad para que los datos de los delincuentes se diluyan en el espacio cibernético. La manipulación de los resultados, por ejemplo, es un delito complejo que puede ser generado a partir de la acción de actores colocados en diferentes jurisdicciones y latitudes, al mismo tiempo, por lo que es muy difícil homogeneizar las acciones de la policía, los marcos de investigación, seguir las pistas de evidencias y pruebas, y establecer juicios y sanciones.

En consecuencia, las tasas de detección y punición continúan siendo muy bajas, mientras los beneficios son elevados. Los deportistas continúan a percibir que, si alguien manipula un juego, las posibilidades de ser descubierto son muy bajas (TIAC, 2014; 2018).

Ante estas evidencias, se torna imperioso:

A. Entender que los fraudes en el mercado de apuestas deportivas, incluyendo aquellos vinculados a la manipulación de competiciones, son una cuestión que traspasa al deporte en sí, y se trata de una cuestión de criminalidad (organizada y no), de delitos financieros y evasión impositiva, de seguridad pública.

B. Percibir la necesidad de procurar legislaciones y regulaciones del mercado a nivel internacional, subsanando las lagunas actuales y definiendo medidas 
concretas para combatir la irregularidad o la ilegalidad en este ámbito comercial.

C. Continuar discutiendo sobre las nuevas éticas del deporte y de las apuestas que se materializan en la nueva coyuntura, incorporando a la discusión análisis de riesgo sobre algunos tipos de apuestas y sobre eventos deportivos que pudiesen no tener las características necesarias para convertirse en objetos "apostables" con la seguridad que debieran.

Este trabajo procuró dar algunas pistas para generar estos ámbitos de discusión, reflexión y creación de más y mejores políticas al respecto. Al mismo tiempo, abre una ventana de oportunidades para continuar estudiando las aristas del fenómeno deporte / apuestas / crimen desde perspectivas multidisciplinares y nuevos enfoques provenientes de la sociología, la ciencia política, la psicología social, la criminalidad y la comunicación.

\section{Agradecimientos}

Este artículo recibió apoyo financiero del Programa Estratégico del Centro de Estudos Internacionais (CEI) del Instituto Universitário de Lisboa (Iscte-IUL) con referencia UIDB/03122/2020. Los autores también agradecen a la Comisión Europea por financiar, mediante el Programa Erasmus + Sport, el proyecto "Training on Protected Reporting System for Professional and Grassroots Sport (T-PREG)", que creo las bases para el trabajo conjunto. Este trabajo, en buena medida, surge del desarrollo y los hallazgos del proyecto T-PREG. Las afirmaciones de este articulo sólo representan la visión de los autores.

\section{Referencias bibliográficas}

Anderson, Jack (2014), "Match fixing and money laundering”, Annual Socio-Legal Studies Association Conference, Robert Gordon University, Aberdeen 9-11 de abril de 2014, accessible online en: https://papers.ssrn.com/sol3/papers.cfm?abstract_id=2424755 (consultado el 8 de mayo de 2020).

Banks, James (2012), “Online gambling and crime: a sure bet?”, The Ethicomp Journal, accessible online en: http://shura.shu.ac.uk/6903/1/Banks_online_gambling.pdf (consultado el 14 de agosto de 2019).

Becker, Gary (1968), “Crime and punishment: an economic approach”, en The economic dimensions of crime, Londres, Palgrave Macmillan, pp. 13-68.

Boniface, Pascal, Sarah Lacarrière, y Pim Verschuuren, (2012), Paris Sportifs et Corruption. Comment Préserver l'Intégrité du Sport, Paris, Colin.

Carpenter, Kevin (2012), "Match-fixing: the biggest threat to sport in the 21st century?", International Sports Law Review, 2 (1), pp. 13-24.

Coubertin, Pierre de (1973 [1908]), Ideario Olimpico. Discursos y Ensayos, Madrid, Instituto Nacional de Educación Física. 
Council of Europe (2014), Council of Europe Convention on the Manipulation of Sports Competitions, accessible online en: https://rm.coe.int/16801cdd7e (consultado el 10 de marzo de 2020).

Dufour, Dany-Robert (2013), “Liberalismo, liberación de las pasiones, pulsiones, tráficos", Diecisiete, 2, pp. 11-27.

European Commission (2007), White Paper on Sport, accessible online en: https://eur-lex.europa.eu/legal-content/EN/TXT/?uri=CELEX\%3A52007DC0391 (consultado en enero de 2020).

European Commission (2011), "Developing the European dimension of sport", accessible online en: https://eur-lex.europa.eu/legal-content/EN/TXT/?uri= CELEX\%3A52011DC0012 (consultado en enero de 2020).

Europol (2017), Serious and Organized Crime Threat Assessment (SOCTA), Bruxelas, European Union, accessible online en: https://www.europol.europa.eu/activities-services/main-reports/serious-and-organ ised-crime-threat-assessment (consultado el 19 de mayo de 2020).

Ferentzy, Peter, y Nigel Turner (2009), “Gambling and organized crime: a review of the literature", Journal of Gambling Issues, 23, p. 119.

FIFPro (2012), Black Book Eastern Europe, SPINS Slovenia, FIFPro, accessible online en: www.lefigaro.fr/assets/pdf/fifpro.pdf (consultado el 18 de mayo de 2020).

Gambling Commission (2016), Gala Coral Group. Failures in Anti-Money Laundering and Social Responsibility Controls Public Statement, UK, Gambling Commission, accessible online en: https://www.gamblingcommission.gov.uk/PDF/ public-statements/Gala-Coral-Public-Statement-April-2016.pdf (consultado el 18 de marzo de 2020).

Gardiner, John A. (1970), The Politics of Corruption. Organized Crime in an American City, Nueva York, Russell Sage Foundation.

Gokhale, Nishant (2009), "Fixing the fixers: the justification of criminal liability for match-fixing", NUJS Law Review, 2 (2), pp. 319-336.

Guía, Diogo (2019), "Apostas desportivas online: regime jurídico do jogo online (RJO) \& manipulação de competições desportivas", Revista de Direito do Desporto, 1, pp. 5-35.

Haller, Mark H. (1971), “Organized crime in urban society: Chicago in the twentieth century", Journal of Social History, 5 (2), pp. 210-234.

Han, Seungbaek (2020), "Match-fixing under the state monopoly sports betting system: a case study of the 2011 K-League scandal", Crime Law and Social Change, DOI: $10.1007 / \mathrm{s} 10611-020-09888-0$

Hill, Declan (2008), The Fix. Soccer and Organized Crime, Toronto, McClelland \& Stewart.

Hill, Declan (2010), "A critical mass of corruption: why some football leagues have more match-fixing than others", International Journal of Sports Marketing E Sponsorship, 11 (3), pp. 38-52.

Hill, Declan (2019), "Sometimes we fixed more games than we could bet", en PlaytheGame, accesible online en: https://www.playthegame.org/news/news-articles/2019/0584_sometimes-we-fixedmore-games-than-we-could-bet/ (consultado el 8 de marzo de 2020).

Huggins, Mike (2018), "Match-fixing: a historical perspective”, The International Journal of the History of Sport, 35 (2-3), pp. 123-140. 
Interpol y IOC (2015), Handbook on Protecting Sport from Competition Manipulation, Lausana, IOC.

IOC y UNODC (2013), Criminalization Approach to Combat Match-Fixing and Illegal/Irregular Betting. A Global Perspective, Lausana / Viena, IOC- UNODC, accessible online en:

https://www.unodc.org/documents/corruption/Publications/2013/Criminalization_ approaches_to_combat_match-fixing.pdf (consultado el 18 de marzo de 2020).

IOC y UNODC (2016), Model Criminal Law Provisions for the Prosecution of Competition Manipulation, Lausanne, IOC, accessible online en:

https://www.unodc.org/documents/corruption/Publications/UNODC-IOC_Model_ Criminal_Law_Provisions_for_the_Prosecution_of_Competition_Manipulation_Bo oklet.pdf (consultado el 20 de marzo de 2020).

IRIS (2017), Preventing Criminal Risks Linked to the Sports Betting Market, informe final del proyecto "New integrated mechanisms for cooperation between public and private actors to identify sports betting risks", patrocinado por la DG Home Affairs of the European Commission, accessible online en: https://library.olympic.org/Default/doc/SYRACUSE/171914/preventing-criminal-ris ks-linked-to-the-sports-betting-market-final-report-june-2017-european-commis?_l g=en-GB (consultado el 19 de mayo de 2020).

Kalb, Christian, y Pim Verschuuren (2013), Money Laundering. The Latest Threat to Sports Betting?, Paris, IRIS Editions.

Kihl, Lisa, James Skinner, y Terry Engelberg (2017) “Corruption in sport: understanding the complexity of corruption", European Sport Management Quarterly, 17 (1), pp. 1-5, DOI: $10.1080 / 16184742.2016 .1257553$.

King, Rufus (1969), Gambling and Organized Crime, Washington, DC, Public Affairs Press.

La Sorbonne / ICSS (2014), Lutter contre la Manipulation des Compétitions Sportives, Paris, Chaire Sorbonne-ICSS.

Maenning, Wolfgang (2005), "Corruption in international sports and sport management: forms, tendencies, extent and countermeasures", European Sport Management Quarterly, 5 (2), pp. 187-225.

Misse, Michel (2007), “Mercados legais, redes de proteção e organização local do crime no Rio de Janeiro", Estudos Avançados, 21 (61), pp. 139-157.

Moriconi, Marcelo (2018a), "The official football match-fixing prevention discourse as a cognitive limitation (the cases of Iberian countries)", Soccer $\mathcal{E}$ Society, 19 (2), pp. 271-287, accessible online en: https://doi.org/10.1080/14660970.2016.1171213 (consultado en enero de 2020).

Moriconi, Marcelo (2018b), “Reframing illegalities: crime, cultural values and ideas of success (in Argentina)", Crime, Law and Social Change, 69 (4), pp. 497-518, DOI: 10.1007/s10611-017-9760-9.

Moriconi, Marcelo (2020), “Deconstructing match-fixing: a holistic framework for sport integrity policies", Crime, Law and Social Change, 74, pp. 1-12, accessible online en: https://doi.org/10.1007/s10611-020-09892-4 (consultado en enero de 2020).

Moriconi, Marcelo, y João Paulo Almeida (2019a), "Portuguese fight against match-fixing: which policies and what ethic?", Journal of Global Sport Management, 4 (1), pp. 79-96, DOI: 10.1080/24704067.2018.1493357. 
Moriconi, Marcelo, y João Paulo Almeida (2019b), “New opportunities for criminal networks: how digital thechnologies are transforming betting industry", en Fabio D'Andrea y Vania Valdi (orgs.), Codice e Luoghi, Meltemi, Italia, pp. 203-223.

Moriconi, Marcelo, y César De Cima, (2020a), “To report, or not to report? From code of silence suppositions within sport to public secrecy realities", Crime, Law and Social Change, 74(1), pp. 55-76, accessible online en: https://doi.org/10.1007/s10611-019-09875-0 (consultado en enero de 2020).

Moriconi, Marcelo, y César De Cima (2020b), “Betting practices among players in Portuguese championships: from cultural to illegal behaviours", Journal of Gambling Studies, 36 (1), pp. 161-181, accessible online en: https://doi.org/10.1007/s10899-019-09880-x (consultado en enero de 2020).

Moriconi, Marcelo, y Rita Teixeira-Diniz (2016), “Mitos, percepciones y actitudes frente a los resultados combinados en Portugal: un estudio con énfasis en los árbitros y los hinchas", Sociologia, Problemas y Práticas, 82, pp. 145-164.

Naciones Unidas (2004), Convención de las Naciones Unidas contra la Delincuencia Organizada Transnacional y Sus Protocolos, Nueva York, Naciones Unidas.

Philippou, Christina (2019), “Towards a unified framework for anti-bribery in sport governance", International Journal of Disclosure and Governance, 16 (2-3), pp. 83-99.

Reuter, Peter (1984), "Police regulation of illegal gambling: frustrations of symbolic enforcement", Annals of the American Academy of Political and Social Science, 474, pp. 36-47.

Sandel, Michael (2012), What Money Can't Buy. The Moral Limits of Markets, Londres, Macmillan.

Sandywell, Barry (2010), “On the globalisation of crime: the internet and new criminality", em Yvonne Jewkes y Majid Yar (orgs.), Handbook of Internet Crime, Cullumpton, Willan, pp. 38-66.

Serby, Tom (2015), “The Council of Europe Convention on Manipulation of Sports Competitions: the best bet for the global fight against match-fixing?", The International Sports Law Journal, 15 (1-2), pp. 83-100.

Soebbing, Brian, Brad Humphreys, y Daniel Mason (2013), “Exploring incentives to lose in professional team sports: do conference games matter?", International Journal of Sport Finance, 8 (3), pp. 192-207.

Spapens, Toine (2017), “Match-fixing”, en Hans Nelen y Dina Siegel (orgs.), Contemporary Organized Crime. Developments, Challenges and Responses, 16, pp. 139-154, Cham, Springer.

Tak, Minhyeok (2018), “Too big to jail: match-fixing, institutional failure and the shifting of responsibility", International Review for the Sociology of Sport, 53 (7), pp. 788-806.

Tak, Minhyeok., Michael P. Sam, y Steven J. Jackson (2018a), “The politics of countermeasures against match-fixing in sport: a political sociology approach to policy instruments", International Review for the Sociology of Sport, 53 (1), pp. 30-48.

Tak, Minhyeok., Michael P. Sam, y Steven J. Jackson (2018b), “The problems and causes of match-fixing: are legal sports betting regimes to blame?", Journal of Criminological Research, Policy and Practice, 4 (1), pp. 73-87.

TIAC (2014), Manipulação de Resultados no Futebol Português. Percepções, Atitudes, Riscos e Narrativas. Informe final del proyecto "Staying on Side. How to Stop Match-Fixing", Lisboa, Transparência e Integridade (TIAC). 
TIAC (2018), Estudo Exploratório, 2018: Perceções e Atitudes dos Atores Desportivos Portugueses sobre Manipulação de Resultados, Informe del proyecto "Anti Match-Fixing Top Training (AMATT)", Lisboa, Transparência e Integridade (TIAC).

UNODC-ICSS (2016), Resource Guide on Good Practices in the Investigation of Match-Fixing, Viena, UNODC, accessible online en: https://www.unodc.org/documents/corruption/Publications/2016/V1602591-RESO URCE_GUIDE_ON_GOOD_PRACTICES_IN_THE_INVESTIGATION_OF_MATC H-FIXING.pdf (consultado el 20 de marzo de 2020).

UNODC y IOC (2019), "Reporting mechanism in sport: a practical guide for development and implementation", Viena, United Nations. Accessible online en: https://www.unodc.org/documents/corruption/Publications/2019/19-09580_Reporti ng_Mechanisms_in_Sport_ebook.pdf (consultado el 20 de marzo de 2020).

Wang, Peng (2012), "The rise of the Red Mafia in China: a case study of organised crime and corruption in Chongqing", Trends in Organized Crime, 16 (1), pp. 49-73.

Zhang, Sheldon X., y Ko-lin Chin (2008), “Snakeheads, mules, and protective umbrellas: a review of current research on Chinese organized crime", Crime, Law, and Social Change, 50 (3), pp. 177-195.

Marcelo Moriconi. Investigador y profesor invitado. Instituto Universitário de Lisboa (Iscte-IUL), Centro de Estudos Internacionais (CEI-Iscte).

Email: marcelo.moriconi@iscte-iul.pt

João Paulo Almeida. Director general. Comité Olímpico de Portugal. Email: jpalmeida@comiteolimpicoportugal.pt

Receção: 31 de março de 2020 Aprovação: 12 de junho de 2020 